\title{
EFFECT OF A CONVECTIVE BOUNDARY CONDITION ON BOUNDARY LAYER SLIP FLOW AND HEAT TRANSFER OVER A STRETCHING SHEET IN VIEW OF THE EXACT SOLUTION
}

\author{
Mona D. Aljoufi, Abdelhalim Ebaid* \\ Department of Mathematics, Faculty of Science, University of Tabuk, \\ P. O. Box 741, Tabuk 71491, Saudi Arabia
}

[Received 24 August 2016. Accepted 12 December 2016]

\begin{abstract}
The exact solutions of a nonlinear differential equations system, describing the boundary layer flow over a stretching sheet with a convective boundary condition and a slip effect have been obtained in this paper. This problem has been numerically solved by using the shooting method in literature. The aim of the current paper is to check the accuracy of these published numerical results. This goal has been achieved via first obtaining the exact solutions of the governing nonlinear differential equations and then, by comparing them with the approximate numerical results reported in literature. The effects of the physical parameters on the flow field and the temperature distribution have been re-investigated through the new exact solutions. The main advantage of the current paper is the simple computational approach that has been introduced to analyze exactly the present physical problem. This simple analytical approach can be further applied to investigate similar problems. Although no remarkable differences have been detected between the current figures and those obtained in literature, the authors believe that if some numerical calculations were available for the fluid velocity and the temperature in literature then the convergence criteria and the accuracy of the shooting method used in Ref. [15] can be validated in view of the current exact expressions.
\end{abstract}

KEY WORDS: Boundary layer; stretching sheet; convective boundary condition; exact solution.

\section{INTRODUCTION}

Many physical models in the applied science are described in the form of linear and nonlinear boundary value problems. In the case of nonlinear boundary value problems, it is usually difficult to obtain the exact analytical solution. Due of this difficulty, most of the researchers in the applied and the engineering sciences directly resort to the numerical methods [1] or the numerical codes to solve their physical models. However, many other authors investigated their physical models by using

*Corresponding author e-mail: aebaid@ut.edu.sa, halimgamil@yahoo.com 
one of the semi-analytical methods such as Adomian decomposition method (ADM) [2-7], differential transformation/Taylor method (DTM) [8-9], homotopy perturbation method (HPM) [10-13], and homotopy analysis method (HAM) [14]. Unfortunately, the accuracy of the numerical solutions derived from these semi-analytical methods cannot be checked without addressing the convergence issue. Another way to check the accuracy of the numerical solutions is to compare them with the exact solutions, if available. It is a well known fact, that the exact solution of any physical model is optimal when available. Such exact solution provides the true behaviour of a given model and avoids putative numerical results. It also serves as a benchmark for any numerical treatment and/or in validating the accuracy of the approximate solutions, derived from the semi-analytical methods of that model.

To ensure the accuracy of these approximate solutions, the convergence issue should be addressed; otherwise, such approximate solutions may lead to incorrect interpretations for the considered model. In this regard, it has been shown recently that the numerical solutions derived from the HPM and the HAM was not correct, when compared with the exact solutions that have been obtained by the authors in [13-14]. Accordingly, the present authors believe that some of the published numerical results should be rechecked through comparisons with the exact solutions after getting them. In order to achieve this goal, we re-investigate the problem studied by Abu Bakar et al. [15], which describes the effect of a convective surface boundary condition on the laminar thermal boundary layer flow past a stretching sheet with partial slip. The boundary layer equations, governed by the partial differential equations were first transformed into the following system of nonlinear ordinary differential equations:

$$
\begin{aligned}
& f^{\prime \prime \prime}(\eta)+f(\eta) f^{\prime \prime}(\eta)-\left(f^{\prime}(\eta)\right)^{2}=0 \\
& \theta^{\prime \prime}(\eta)+\operatorname{Pr} f(\eta) \theta^{\prime}(\eta)=0
\end{aligned}
$$

where $\eta$ is the similarity variable, $\operatorname{Pr}$ is Prandtl number, $f(\eta)$ is the stream function, and $\theta(\eta)$ is the temperature distribution of the boundary layer. Details of PDEs describing the flow and the similarity transformations can be found in [15], they were not mentioned here to avoid repetition. The flow is a subject to the following boundary conditions:

$$
\begin{aligned}
& f(0)=0, \quad f^{\prime}(0)=1+K f^{\prime \prime}(0), \quad f^{\prime}(\infty)=0, \\
& \theta^{\prime}(0)=-\gamma[1-\theta(0)], \quad \theta(\infty)=0,
\end{aligned}
$$

where $K$ is the slip parameter and $\gamma$ is the convective parameter. The method of obtaining the exact solutions is discussed in the next section. The objectives of the present paper can be summarized as follows: 
1) Obtaining the exact solutions for the system of nonlinear differential equations governing the flow.

2) The obtained exact solutions are used to introduce various plots for the exact temperature and the exact velocity.

3) The final task is to compare the current exact results for the velocity and the temperature with those approximately obtained, using the shooting method in Ref. [15].

\section{EXACT SOLUTIONS}

Firstly, it should be noted that the current system of nonlinear equations is not coupled. This means, that if we are able to solve exactly the $f$-equation (1) with the boundary conditions (3), then we will be able to solve the $\theta$-equation analytically. Moreover, the exact solution of the $f$-equation (1) with the boundary conditions (3) is not a difficult task, since it was available in many previous studies, such as [16]. In order to solve the system (1-4), we usually start with Eq. (1) in which the function $f(\eta)$ is the only unknown. The following assumption is used:

$$
f(\eta)=a+b \mathrm{e}^{-\beta \eta},
$$

where $\beta$ should be positive, so that the infinity boundary condition for $f(\eta)$ is satisfied. Solution in the form (5) is a specific solution of the Eq. (1). On using Eq. (5) into Eq. (1), we have

$$
b \beta^{2} \mathrm{e}^{-\beta \eta}(a-\beta)=0,
$$

which leads to

$$
a=\beta .
$$

Applying the first two boundary conditions given in (3), we obtain

$$
\begin{gathered}
a+b=0, \\
K b \beta^{2} b \beta+1=0 .
\end{gathered}
$$

In view of Eqs. (8-9), we obtain $a$ and $b$ in terms of $\beta$ as

$$
b=-\beta,
$$

where $\beta$ satisfies the cubic-algebraic equation

$$
K \beta^{3}+\beta^{2}-1=0 .
$$


Therefore, $f(\eta)$ is given by

$$
f(\eta)=\beta\left(1-\mathrm{e}^{-\beta \eta}\right)
$$

where $\beta$ is a positive real root of Eq.(11). Substituting $f(\eta)=\beta\left(1-\mathrm{e}^{-\beta \eta}\right)$ into Eq.

(2), we have the following differential equation in only $\theta(\eta)$ :

$$
\theta^{\prime \prime}(\eta)+\operatorname{Pr} \beta\left(1-\mathrm{e}^{-\beta \eta}\right) \theta(\eta)=0,
$$

Integrating the last equation once w.r.t $\eta$ from 0 to $\eta$ yields

$$
\theta(\eta)=\theta^{\prime}(0) \mathrm{e}^{P r} \times \mathrm{e}^{-P r\left(\beta \eta-\mathrm{e}^{-\beta \eta}\right)},
$$

Integrating Eq. (14) once again w.r.t $\eta$ from 0 to $\eta$, we obtain

$$
\theta(\eta)=\theta(0)+\theta^{\prime}(0) \mathrm{e}^{P r} \times \int_{0}^{\eta} \mathrm{e}^{-\operatorname{Pr} \beta \sigma} \times \mathrm{e}^{-P r e^{-\beta \sigma}} d \sigma,
$$

or

$$
\theta(\eta)=\theta(0)-\gamma[1-\theta(0)] \mathrm{e}^{P r} \times I(\eta)
$$

where

$$
I(\eta)=\int_{0}^{\eta} \mathrm{e}^{-\operatorname{Pr} \beta \sigma} \times \mathrm{e}^{-P r e^{-\beta \sigma}} d \sigma .
$$

In order perform the integration in the right hand side we use the assumption

$$
\mu=\operatorname{Pre}^{-\beta \sigma}
$$

Accordingly, we have:

$$
d \sigma=-\frac{d \mu}{\beta \mu}, \quad \mathrm{e}^{-\beta \sigma}=\frac{\mu}{\operatorname{Pr}} .
$$

On using the definition of the incomplete gamma function

$$
\Gamma\left(c, z_{0}, z_{1}\right)=\int_{z_{0}}^{z_{1}} \mu^{c-1} \mathrm{e}^{-\mu} d \mu
$$


then, the integral in (17) becomes

(21) $I(\eta)=\frac{1}{\beta(P r)^{P r}} \int_{P r \mathrm{e}^{-\beta \sigma}}^{P r} \mu^{P r-1} \mathrm{e}^{-\mu} d \mu=\frac{1}{\beta(\operatorname{Pr})^{P r}} \Gamma\left(\operatorname{Pr}, \operatorname{Pr} \mathrm{e}^{-\beta \eta}, \operatorname{Pr}\right)$.

Therefore, we have from Eq. (16) that

$$
\theta(\eta)=\theta(0)-\gamma[1-\theta(0)] \frac{e^{P r}}{\beta(P r)^{P r}} \Gamma\left(\operatorname{Pr}, \operatorname{Pr} e^{-\beta \eta}, \operatorname{Pr}\right)
$$

Applying the infinity boundary condition $\theta(\infty)=0$, yields

$$
0=\theta(0)-\gamma[1-\theta(0)] \frac{\mathrm{e}^{P r}}{\beta(P r)^{P r}} \Gamma\left(\operatorname{Pr}, \operatorname{Pr} e^{-\beta \eta}, \operatorname{Pr}\right) .
$$

On solving the last equation for $\theta(0)$, we obtain

$$
\theta(0)=\frac{1}{1+\frac{1}{\gamma \Omega}}
$$

where

$$
\Omega=\frac{\mathrm{e}^{P r}}{\beta(\operatorname{Pr})^{P r}} \Gamma(\operatorname{Pr}, 0, \operatorname{Pr}) .
$$

Inserting the value of $\theta(0)$ into Eq. (22), we get

$$
\theta(\eta)=\frac{\gamma \Omega}{1+\gamma \Omega}\left[1-\frac{\Gamma\left(\operatorname{Pr}, \operatorname{Pr} e^{-\beta \eta}, \operatorname{Pr}\right)}{\Gamma(\operatorname{Pr}, 0, \operatorname{Pr})}\right]
$$

This expression for $\theta(\eta)$ can be further simplified by using the following identity for the incomplete gamma function:

$$
\Gamma\left(c, 0, z_{1}\right)-\Gamma\left(c, z_{0}, z_{1}\right)=\Gamma\left(c, 0, z_{0}\right) .
$$

Hence, the exact solution for the temperature distribution $\theta(\eta)$ is finally given by

$$
\theta(\eta)=\frac{\gamma \Omega}{1+\gamma \Omega}\left[\frac{\Gamma\left(\operatorname{Pr}, 0, \operatorname{Pr} e^{-\beta \eta}\right)}{\Gamma(\operatorname{Pr}, 0, \operatorname{Pr})}\right]
$$


where $\Omega$ is already defined by Eq. (25). The exact solutions, given by (12) and (28), can be easily checked by direct substitution in the system (1-4). Further, in the absence of the convective boundary condition, i.e, as $\gamma \rightarrow \infty$, we obtain

$$
\begin{aligned}
\theta(\eta) & =\left[\frac{\Gamma\left(\operatorname{Pr}, 0, P r e^{-\beta \eta}\right)}{\Gamma(\operatorname{Pr}, 0, P r)}\right] \lim \gamma \rightarrow \infty \frac{\gamma \Omega}{1+\gamma \Omega} \\
& =\left[\frac{\Gamma\left(\operatorname{Pr}, 0, P r e^{-\beta \eta}\right)}{\Gamma(\operatorname{Pr}, 0, \operatorname{Pr})}\right] \lim _{\gamma \rightarrow \infty} \frac{\Omega}{\frac{1}{\gamma}+\Omega} \\
& =\frac{\Gamma\left(\operatorname{Pr}, 0, \operatorname{Pr} e^{-\beta \eta}\right)}{\Gamma(\operatorname{Pr}, 0, \operatorname{Pr})},
\end{aligned}
$$

which agrees with the exact solution obtained very recently by Aly and Ebaid [16] (Eq. 33), in the case of no suction/injection, i.e., $s=0$ and at $\lambda=1$ and $\tau=1 / \operatorname{Pr}$, see Ref. [16] for details.

\section{DISCUSSION}

The equations with boundary conditions (1-4) could be solved by semi-analytical methods. The authors may suggest the readers to do this in future works and compare their results with the current exact solutions. In this respect, the authors recommend the readers to revise Refs [17-21], that show how to use semi-analytical methods for different types of ordinary differential equations. The nonlinear ordinary differential equations (1) and (2), subjected to the boundary conditions (3-4), were solved exactly in the previous section, using a simple but effective analytical procedure. The present numerical computations have been carried out for various values of the parameters involved, namely Prandtl number $\mathrm{Pr}$, slip parameter $K$ and convective parameter

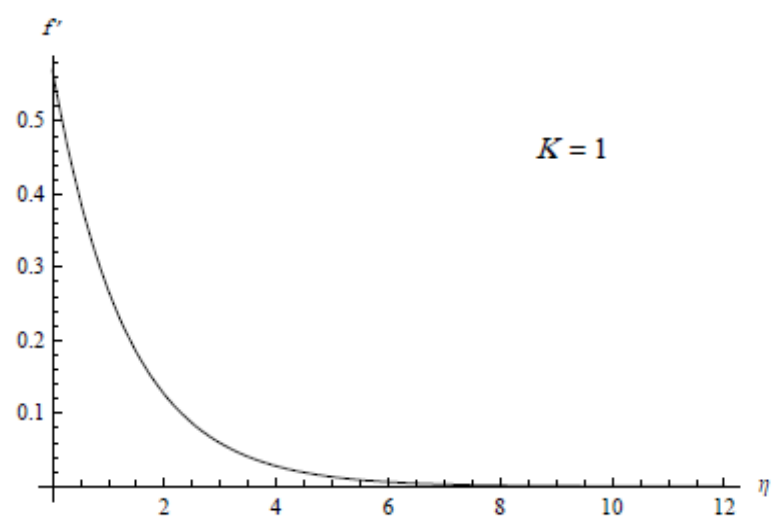

Fig. 1. Velocity profiles for $K=1$. 


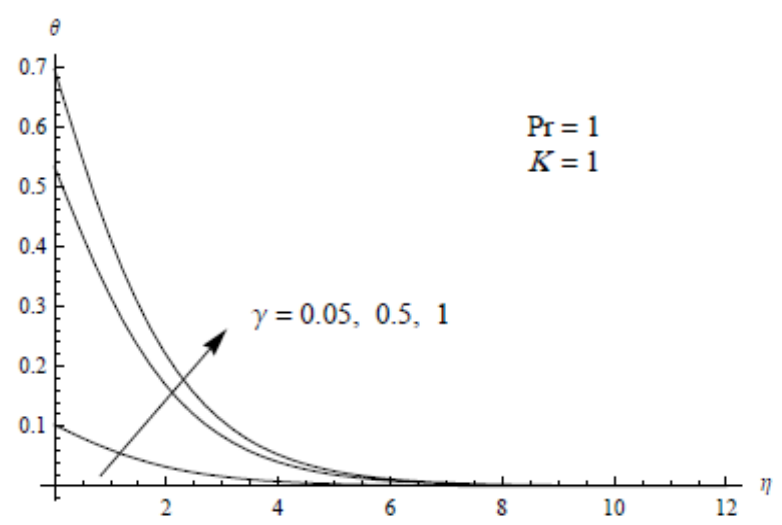

Fig. 2. Temperature profiles for different values of $\gamma$.

$\gamma$ at the same data, that have been considered in [15]. It is clear from Eqs. (1112), that the convective parameter $\gamma$ and the Prandtl number $P r$ give no effect to the flow field. For purpose of comparison with the results obtained in [15], we have plotted the velocity field at $K=1$ in Fig. 1 . There is no remarkable difference between Fig. 1 of the current study and Fig. 1 obtained in [15]. However, if some numerical calculations were available for the velocity of the fluid in Ref. [15], then the convergence criteria for the shooting method can be validated in view of the current exact velocity expression. In this regard, the authors of the present paper believe that there are some numerical errors, which cannot be estimated through only graphs and without re-calculating the numerical values of the velocity by the shooting method at some chosen values of the similarity variable.

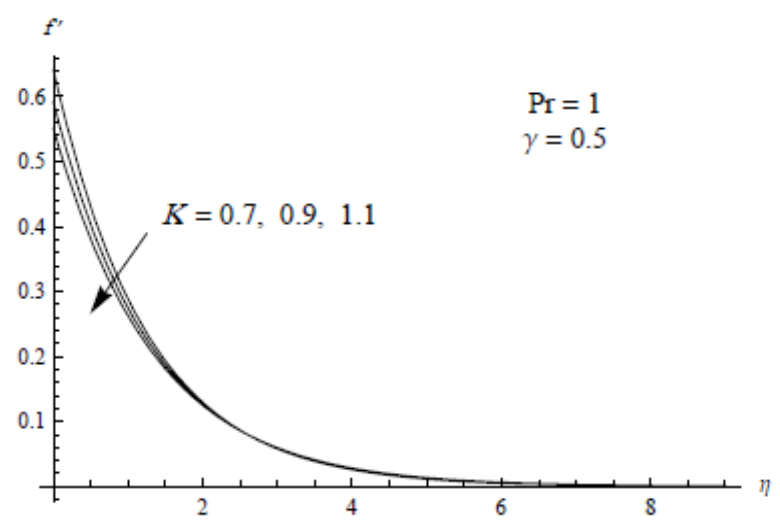

Fig. 3. Velocity profiles for different values of $K$. 


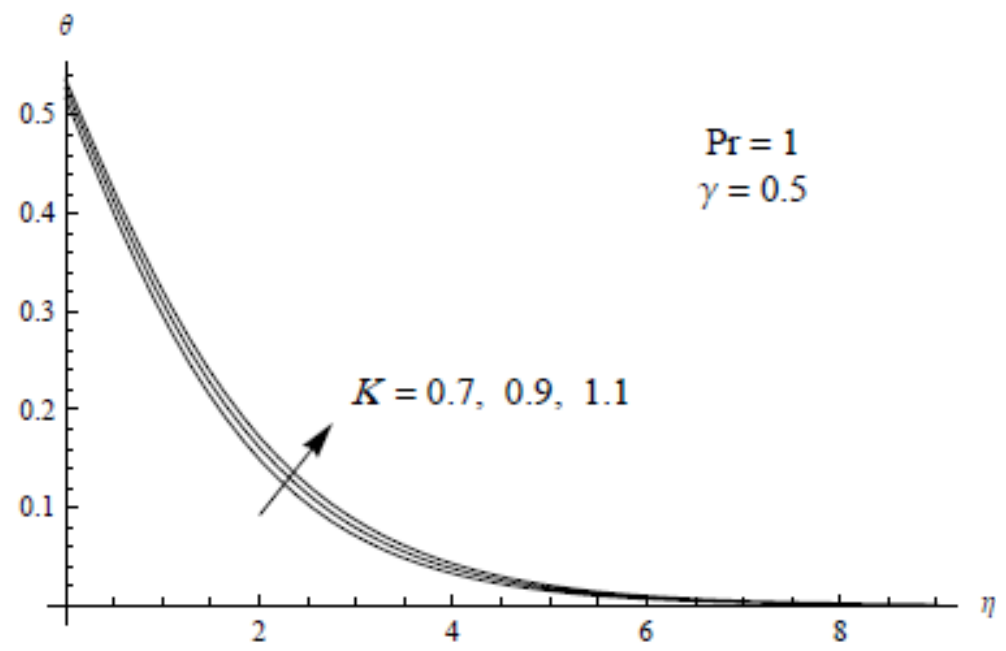

Fig. 4. Temperature profiles for different values of $K$.

The impact of the convective parameter $\gamma$ on the temperature field at $\operatorname{Pr}=K=1$ is depicted in Fig. 2. It is observed from this figure, that increasing $\gamma$ increases both the temperature at the surface, i.e., $\theta(0)$ and the temperature gradient at the surface $\theta^{\prime}(0)$ (in absolute sense), this conclusion is also reported in [15]. The effects of the slip parameter $K$ on the velocity and temperature profiles are illustrated in Figs. 3

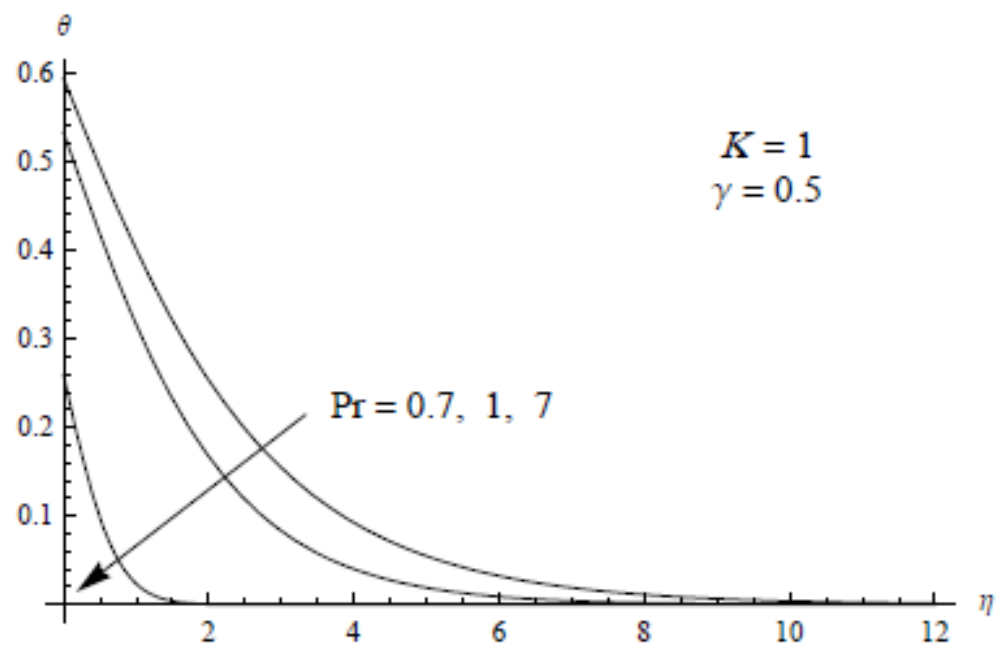

Fig. 5. Temperature profiles for different values of $\operatorname{Pr}$. 
and 4, respectively, both for $\operatorname{Pr}=1$ and $\gamma=0: 5$. It is seen in Fig. 3, that the velocity gradient at the surface $f^{\prime \prime}(0)$ decreases as $K$ increases. Thus and as reported in [15], the skin friction coefficient decreases in the presence of slip at the boundary and is inversely proportional to the magnitude of the slip. It can be seen in Fig. 4, that the temperature increases with increasing the slip parameter $K$. The effect of Prandtl number on the temperature distribution is displayed in Fig. 5, at $K=1$ and $\gamma=0: 5$. It is also observed from this figure, that the temperature gradient at the surface increases as $P r$ increases, which implies an increase in the heat transfer rate at the surface. The main observation here is the coincidence between the published approximate numerical solutions obtained in [15] and the current exact solutions. This may refer to the effectiveness of the shooting method that has been used as a numerical method of solution in Ref. [15]. This paper is basically of mathematical nature according to the aim hence, the physical argumentation was shorted.

\section{CONCLUSiOns}

The system of nonlinear differential equations, describing the boundary layer flow over a stretching sheet with a convective boundary condition and slip effect has been exactly solved in this paper. The effect of the physical parameters on the flow field and the temperature distribution has been re-investigated through the new exact solutions. No remarkable differences have been detected between the current exact results and those approximately obtained via the shooting method in [15]. However, one of the main objectives of the current paper was to present a simple analytical procedure to obtain the exact solutions of the considered system. This goal has been achieved and consequently the comparisons have been made between the present results and those published in literature. This simple analytical procedure may be used and extended for other similar physical problems.

\section{ACKNOWLEDGMENT}

The authors would like to acknowledge financial support for this work from the Deanship of Scientific Research (DSR), University of Tabuk, Tabuk, Saudi Arabia, under Grant no. S-023-1437.

\section{REFERENCES}

[1] Aly, E. H., A. EbAid. New Analytical and Numerical Solutions for mixed Convection Boundary-layer Nanofluid Flow along an Inclined Plate Embedded in a Porous Medium. Journal of Applied Mathematics, Volume (2013), Article ID 219486, 7 pages, http://dx.doi.org/10.1155/2013/219486.

[2] Adomian, G. Solving Frontier Problems of Physics: The Decomposition Method, Boston, Kluwer. Acad., 1994. 
[3] EBAID, A. Approximate Analytical Solution of a Nonlinear Boundary Value Problem and its Application in Fluid Mechanics. Z. Naturforsch A, 66 (2011), 423-426.

[4] EBAID, A. A New Analytical and Numerical Treatment for Singular Two-point Boundary Value Problems via the Adomian Decomposition Method. J. Comput. Appl. Math., 235 (2011), 1914-1924.

[5] Ali, E. H., A. EBAID, R. RACH. Advances in the Adomian Decomposition Method for Solving Two-point Nonlinear Boundary Value Problems with Neumann Boundary Conditions. Comput. Math. Applic., 63 (2012), 1056-1065.

[6] Wazwaz, A.-M., R. RaCh, J.-S. Duan. Adomian Decomposition Method for Solving the Volterra Integral Form of the Lane-Emden Equations with Initial Values and Boundary Conditions. Appl. Math. Comput., 219 (2013), No. 10, 5004-5019.

[7] EBAID, A. Analytical Solutions for the Mathematical Model Describing the Formation of Liver Zones via Adomian's Method. Computational and Mathematical Methods in Medicine, Volume 2013, Article ID 547954, 8 pages.

[8] EBAID, A. Approximate Periodic Solutions for the Non-linear Relativistic Harmonic Oscillator via Differential Transformation Method. Commun. Nonlin. Sci. Numer. Simulat., 15 (2010), 1921-1927.

[9] EBAID, A. A Reliable Aftertreament for Improving the Differential Transformation Method and its Application to Nonlinear Oscillators with Fractional Nonlinearities. Commun. Nonlin. Sci. Numer. Simulat., 16 (2011), 528-536.

[10] HE, J.-H. Homotopy Perturbation Technique. Computer Methods in Applied Mechanics and Engineering, 178 (1999), No. 3-4, 257-262.

[11] ArIEL, P. D. The Three-dimensional Flow Past a Stretching Sheet and the Homotopy Perturbation Method. Computers \& Mathematics with Applications, 54 (2007), No. 7-8, 920-925.

[12] Aly, E. H., A. EBAid. New Analytical and Numerical Solutions for Mixed Convection Boundary Layer Nanofluid Flow along an Inclined Plate Embedded in a Porous Medium. Journal of Applied Mathematics, 2013, Article ID 219486, http://dx.doi.org/10.1155/2013/219486.

[13] EBAID, A. Remarks on the Homotopy Perturbation Method for the Peristaltic Flow of Jeffrey Fluid with Nano-particles in an Asymmetric Channel. Computers \& Mathematics with Applications, 68 (2014), 7785.

[14] Ebaid, A., S. H. Alatawi. Influence of Wall Properties on the Peristaltic Flow of a Nanofluid in View of the Exact Solutions: Comparisons with Homotopy Analysis Method. Z. Naturforsch A, 69 (2014), 199-206.

[15] Abu Bakar, N. A., W. M. K. A. W. Zaimi, R. Abdul Hamid, B. Bidin, A. ISHAK. Boundary Layer Flow over a Stretching Sheet with a Convective Boundary Condition and Slip Effect. World Applied Sciences Journal, 17 (2012), 49-53.

[16] Aly, E. H., A. EBAID. Exact Analytical Solution for Suction and Injection Flow with Thermal Enhancement of Five Nanofluids over an Isothermal Stretching Sheet with Effect of the Slip Model: A Comparative Study. Abstract and Applied Analysis, Volume, 2013, Article ID 721578, 14, pages, http://dx.doi.org/10.1155/2013/721578. 
[17] Hajmohammadi, M. R., S. S. Nourazar, A. H. Manesh. Semi-analytical Treatments of Conjugate Heat Transfer. Journal of Mechanical Engineering Science, 227 (2012), 492-503.

[18] Hajmohammadi, M. R., S. S. Nourazar. On the Solution of Characteristic Value Problems arising in Linear Stability Analysis; Semi Analytical Approach. Applied Mathematics \& Computation, 239 (2014), 126-132.

[19] Hajmohammadi, M. R., S. S. Nourazar. Conjugate Forced Convection Heat Transfer from a Heated Flat Plate of Finite Thickness and Temperature Dependent Thermal Conductivity. Heat Transfer Engineering, 35 (2014) 863-874.

[20] Hajmohammadi, M. R., S. S. Nourazar. On the Insertion of a Thin Gas Layer in Micro Cylindrical Couette Flows Involving Power Law Liquids. International Journal of Heat \& Mass Transfer, 75 (2014), 97-108.

[21] Hajmohammadi, M. R., S. S. Nourazar, A. Campo. Analytical Solution for Two Phase Flow between Two Rotating Cylinders Filled with Power Law Liquid and a Micro Layer of Gas. Journal of Mechanical Science and Technology, 28 (2014), No. 5, 1849-1854, DOI: 10.1007/s122060130913y. 